Musées, Patrimoine et Culture scientifiques et techniques

$169 \mid 2017$

janvier-février 2017

\title{
Les matériaux de mise en forme des spécimens naturalisés
}

\section{Gaëlle Duffau}

\section{(2) OpenEdition \\ Journals}

Édition électronique

URL : http://journals.openedition.org/ocim/1734

DOI : $10.4000 /$ ocim. 1734

ISSN : 2108-646X

Éditeur

OCIM

Édition imprimée

Date de publication : 1 janvier 2017

Pagination : 19-25

ISSN : 0994-1908

Référence électronique

Gaëlle Duffau, «Les matériaux de mise en forme des spécimens naturalisés », La Lettre de l'OCIM [En ligne], 169 | 2017, mis en ligne le 01 janvier 2018, consulté le 19 avril 2019. URL : http:// journals.openedition.org/ocim/1734; DOI : 10.4000/ocim.1734

Ce document a été généré automatiquement le 19 avril 2019

Tous droits réservés 


\title{
Les matériaux de mise en forme des spécimens naturalisés
}

\author{
Gaëlle Duffau
}

Étapes du montage traditionnel d'un spécimen naturalisé

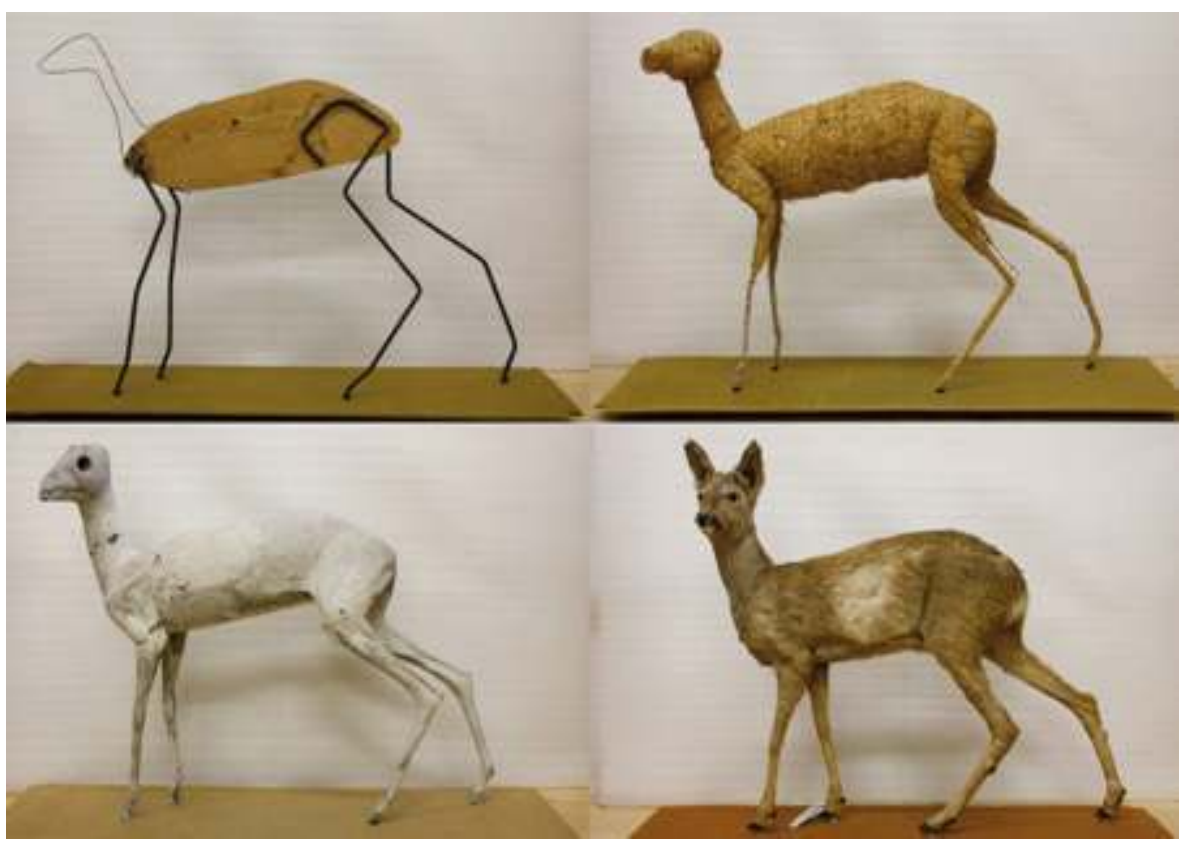

(c) HECR Arc, G.B, 2015

1 La taxidermie utilise de nombreux matériaux d'origines diverses. Outre la peau, les os et les phanères, un spécimen naturalisé est un assemblage d'une structure en bois ou en métal (anciennement en os), d'un rembourrage végétal, minéral ou synthétique, et de matériaux de finition (du verre ou une résine synthétique pour les yeux, de la peinture pour les retouches, et parfois du plâtre ou de l'argile pour le museau et les oreilles). Nous parlons ainsi d'objet composite complexe dont chaque matériau réagira différemment au vieillissement ou à son environnement. 
2 Au cours de l'histoire, de nombreuses évolutions techniques ont eu lieu, souvent accompagnées de l'apparition de nouveaux procédés de mise en forme et de nouvelles matières. Le rembourrage, qui représente souvent le plus grand volume d'un spécimen, est également le plus méconnu de par son emplacement interne et caché. Ainsi, il est impossible de connaître tous les matériaux utilisés depuis le XVII ${ }^{\mathrm{e}}$ siècle en raison de leur très grand nombre, du manque de documentation écrite (la transmission des connaissances étant essentiellement orale) et du coté expérimental de la pratique de la taxidermie. De tous ces matériaux, nous connaissons seulement ceux figurant dans les quelques écrits existants ou qui nous sont parvenus. Une grande partie a en effet disparu car ils ne permettaient pas la conservation du spécimen sur le long terme.

Aujourd'hui, le manque de connaissances sur les matériaux de rembourrage utilisés peut compliquer la conservation des Naturalia. Sur certains spécimens, les dégradations physiques observées ont pour origine des variations dimensionnelles du matériau interne (déchirures, affaissements de la peau...).

Armature de bois du montage du buffle de Jules Terrier au XIX ${ }^{\mathrm{e}}$ siècle

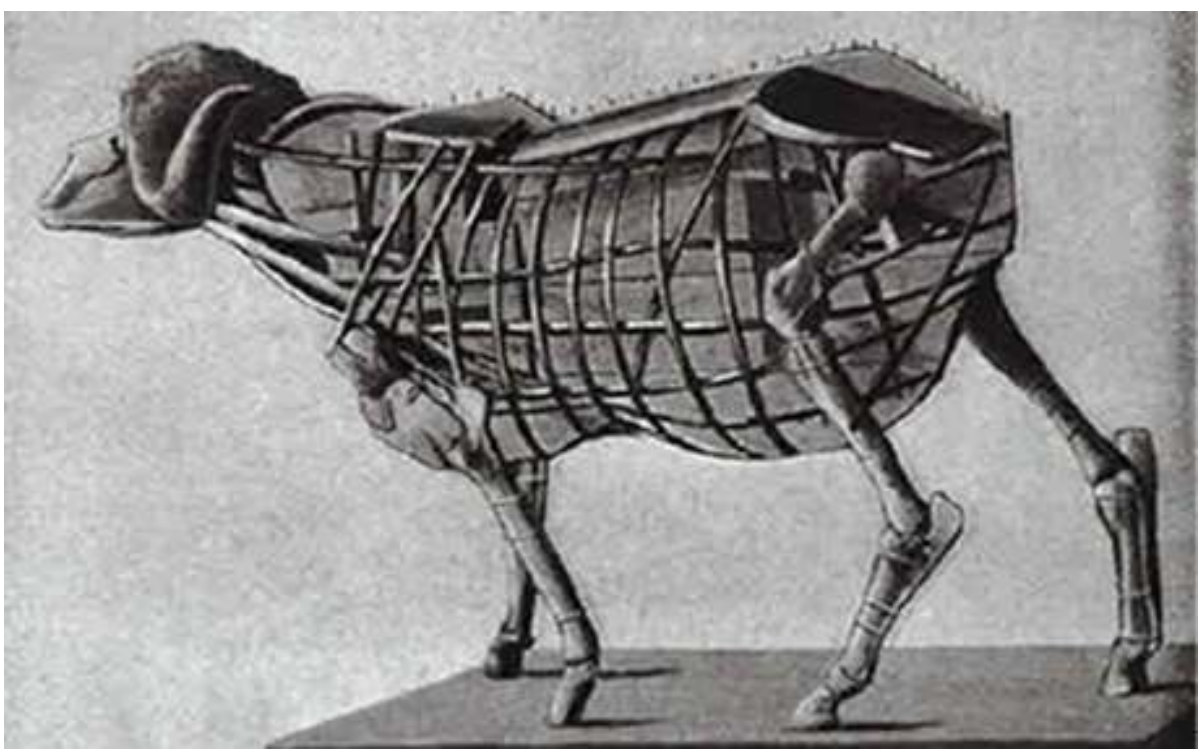

(c) Didier et Boudarel, 1921

\section{Évolution de la mise en forme des spécimens}

Le montage le plus pratiqué (dit traditionnel) implique la mise en forme de la peau tannée autour d'une structure qui recrée le volume de l'animal. Cette structure combine souvent plusieurs matériaux de rembourrage utilisés selon leurs caractéristiques et fixés autour d'une âme rigide (os, fer, bois). La technique de fabrication du mannequin a évolué au cours de l'histoire, impliquant une modification de mise en forme et de matériaux dans le but d'améliorer la conservation du spécimen, le réalisme de l'animal et de faciliter le façonnage. Cette évolution peut se diviser en trois grandes périodes, entraînant chacune l'apparition de nouveaux types de matériaux. 


\section{XVII'-XVIII'e siècles : "l'empaillage"}

5 Les premières sources sur les méthodes de mise en forme nous indiquent qu'au XVII siècle, le volume de l'animal est réalisé par remplissage (ou bourrage) de la peau avec des matières souples, essentiellement d'origine végétale, ce qui donne souvent un mannequin difforme et peu réaliste. La majorité des réalisations de cette époque ont disparu à cause des matériaux de mise en forme mais également en raison de l'absence systématique de tannage.

6 Au XVIII ${ }^{\mathrm{e}}$ siècle, le mannequin se construit par l'assemblage des os longs de l'animal, du crâne et d'un matériau de rembourrage. Le volume est alors réalisé avec un végétal à fibres longues, travaillé sous forme de filasse ou d'étoupe, enroulé, coupé, ou haché pour remplir le spécimen. Le détail des lèvres, des oreilles, et le tour des yeux est fait avec un matériau déformable de type pâte à modeler, mastic, ou argile ${ }^{1}$. Malgré un mannequin plus "construit", la pratique reste très artisanale et ne permet pas une conservation à long terme.

\section{$\mathrm{XIX}$ siècle : perfectionnement des mannequins}

7 La technique évolue au XIX ${ }^{e}$ siècle grâce à Jules Terrier, taxidermiste du Muséum de Paris qui conçoit le mannequin comme une sculpture rigide. Les os sont remplacés par une armature en baguettes de bois et en tiges de fer pour recréer le profil de l'animal et les pattes. Ceci permet d'avoir un spécimen plus léger, qui se conserve mieux (en diminuant les problèmes d'acides gras), mais permet également d'avoir un squelette complet pour les collections ostéologiques. Les volumes sont rendus par des superpositions de fibres végétales travaillées en fuseau puis recouvertes de plâtre mélangé à du foin ou des fibres textiles pour recréer le détail des muscles. La matière est ainsi ajoutée jusqu'à l'obtention d'un volume satisfaisant et la peau est collée sur la dernière couche de plâtre. Les volumes des lèvres et du contour des yeux sont reproduits à l'aide d'un matériau modelable, et les yeux en verre, en émail ou en résine sont ensuite placés sur l'ensemble.

8 Le XIX ${ }^{\mathrm{e}}$ siècle est l'âge d'or de la taxidermie, de l'explosion des cabinets de naturalisation, et des écris techniques. Les spécimens sont fabriqués à la chaîne avec ce même protocole (encore pratiqué par certains taxidermistes actuels), et la généralisation de l'utilisation du savon arsenical permet une bien meilleure conservation ${ }^{2}$. Mais, dans un but de perfectionnement de la forme, c'est également une période d'expérimentation des matériaux, ce qui entraîne une très grande variété de rembourrages végétaux (plus ou moins adaptés) ainsi que l'apparition des rembourrages minéraux.

\section{$X X{ }^{e}-X X I^{e}$ siècles : révolution du synthétique}

9 Avec l'apparition des matériaux synthétiques au milieu du XXe siècle, la taxidermie change une nouvelle fois de visage. Ces matériaux sont utilisés dans ce domaine depuis 1960. Ces matériaux imputrescibles, non hygroscopiques et qui n'attirent pas les insectes semblent résoudre tous les problèmes de conservation des spécimens (du moins en apparence) et facilitent la mise en forme. Le mannequin est alors réalisé par enlèvement de matière comme le fait un sculpteur sur la pierre, donnant un volume plein et rigide tout en restant léger. Il est travaillé à la scie, à la râpe ou au rifloir, et poncé au papier de 
verre avant d'être recouvert de la peau. Certains mannequins sont usinés à la chaîne et vendus déjà prêts à l'emploi, ne laissant au taxidermiste que les étapes de l'adaptation aux dimensions de la peau et des finitions.

En parallèle, les mannequins végétaux sont encore utilisés, mais pour diminuer les variations dimensionnelles certains taxidermistes (en France et en Suisse) trempent le corps artificiel dans une résine synthétique, de la colle, de la gomme laque, ou de la dextrine $^{3}$. Cette pratique disparaît progressivement à la fin du XX $X^{e}$ siècle.

11 Les matériaux synthétiques sont révolutionnaires mais avec le temps, beaucoup se sont révélés chimiquement instables, provoquant de nombreux problèmes de conservation. Certains se dégradent très vite et entraînent des dégagements acides et des pertes de cohésion importante, pouvant conduire à la perte totale du spécimen. De sorte que, si la durée de fabrication s'est nettement améliorée, l'apparition de ces matériaux a également conduit à l'accélération des dégradations des spécimens.

12 Aujourd'hui, les mannequins synthétiques se sont démocratisés, et malgré le manque de recul sur la durabilité des matériaux, leur facilité de mise en forme fait l'unanimité dans la profession.

\section{Référencement des matériaux de rembourrage}

13 Les matériaux de rembourrage sont utilisés pour remplacer les tissus mous de l'animal qui ont été retirés pour la conservation du spécimen. Historiquement, ce sont les premiers matériaux à avoir été ajoutés à l'animal. À l'origine, ils avaient pour but d'absorber les fluides corporels et faciliter le séchage de la peau, puis lorsque le spécimen est devenu plus réaliste, le rembourrage a eu pour fonction de donner son volume au spécimen. Ils doivent être soit modelables, soit déformables, sans structure rigide propre pour leur donner la forme voulue, et sont choisis par les praticiens selon le type de montage, la taille du spécimen et leurs préférences personnelles.

Durant toutes les époques, les préparateurs ont été à la recherche d'une matière recréant avec finesse les volumes du corps de l'animal, et n'apportant pas une nouvelle source de dégradation au spécimen. La grande variété des rembourrages utilisés, et le manque de documentation de la part des praticiens font qu'il est presque impossible d'en dresser une liste exhaustive. Durant ces recherches, nous avons pu constater le manque de précision dans les écrits des taxidermistes. Jusqu'au XVIII ${ }^{\mathrm{e}}$ siècle, les termes employés (comme étoupe ou filasse par exemple) dans les manuels ne renseignent pas sur la nature exacte du matériau. Ce n'est qu'au XIX ${ }^{e}$ siècle que l'on trouve un vocabulaire plus pertinent et diversifié.

15 Ainsi, le recensement réalisé s'appuie sur plusieurs sources bibliographiques (voir bibliographie) dont une recherche menée par Williams et Hawks en 1987 aux États-Unis qui ont recensé 36 familles de matériaux de rembourrages différents utilisés par des praticiens anglo-saxons sur une période allant $d u X V I I^{e}$ au XXe siècle. Nous avons également combiné les résultats d'autres recherches sur les matériaux de mise en forme des spécimens avec des manuels de taxidermie de différentes époques et des témoignages de taxidermistes ou de spécialistes du domaine de l'histoire naturelle. Enfin, cette recherche documentaire a été complétée par l'identification des rembourrages d'un corpus de 20 spécimens (mammifères, oiseaux et reptiles) d'origines et de tailles variées conservés au muséum d'Histoire naturelle de Neuchâtel en Suisse. L'identification a en 
effet permis de mettre au jour de nouveaux matériaux qui ne figuraient pas dans les recherches documentaires.

Les rembourrages principaux identifiés durant ce travail sont présentés ci-dessous, classés selon leur origine végétale, animale, minérale ou synthétique, et l'intégralité des résultats de la recherche est disponible dans le tableau final.

Finitions d'un mannequin de mouflon par Jack Thiney (MNHN) à la râpe, rifloir, et papier de verre pour lisser la surface.

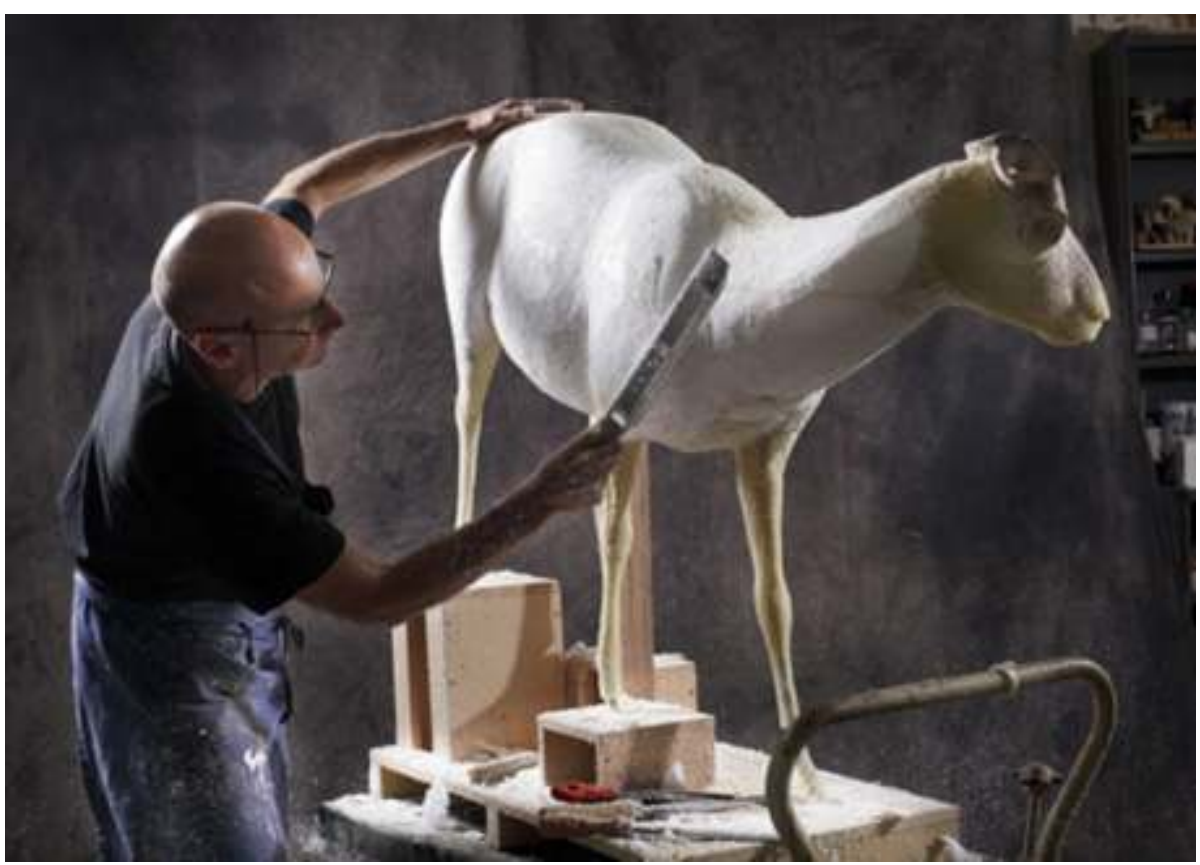

(C) Vekemans, 2014

\section{Les matériaux d’origine végétale}

Les rembourrages les plus anciens (les premiers témoignages d'utilisation en taxidermie de ce procédé remontent au début du XVII ${ }^{e}$ siècle) et les plus utilisés au cours des siècles sont les matériaux cellulosiques. Ils peuvent être classés en plusieurs sous-catégories en fonction de leur forme et de leurs caractéristiques : les fibres textiles, les végétaux en vrac, les tiges (ou chaume), les dérivés du papier et du textile, et les dérivés du bois.

\section{Les fibres textiles.}

Les premiers matériaux sont probablement les fibres de lin et de chanvre hachées pour les petits spécimens ou entières pour les plus grands. Avec le coton, utilisé essentiellement pour les oiseaux, ce sont les rembourrages les plus communs quelles que soient l'époque et la région géographique. Cependant le coton a été critiqué par certains praticiens car il a tendance à déformer le spécimen et à perdre son élasticité avec le temps ${ }^{4}$. Williams et Hawks (en 1987) ont recensé plus de 50 témoignages d'utilisation de ces trois matériaux, et lors de notre étude de cas, $1 / 3$ des matériaux identifiés étaient des fibres textiles. 


\section{Les végétaux en vrac.}

19 De façon moins fréquente, des petits végétaux ou des fragments de végétaux, de taille et de forme hétérogènes, sont observables dans des spécimens, disposés sans organisation particulière, telles que les mousses végétales. Elles sont légères et volumineuses et donc utilisées pour le remplissage de grands oiseaux et de certains mammifères, mais elles sont également particulièrement hygroscopiques et peuvent provoquer des gonflements. Nous pouvons également considérer que la tourbe fait partie des végétaux en vrac dégradés. Elle a été utilisée jusqu'au début du XXI ${ }^{\text {e }}$ siècle en France et en Suisse. Elle est sculptée à la forme du corps et parfois trempée dans de la paraffine chaude afin de diminuer les variations dimensionnelles 5 .

\section{Les tiges/chaume.}

Dans la catégorie des tiges, figurent le foin, la paille (famille des graminées) ou des roseaux pris en bord d'étangs. Par leur caractéristique rustique, ils donnent une grande solidité aux mannequins et ont donc été privilégiés pour les grands spécimens. Des témoignages de préparateurs en bord de mer attestent de l'utilisation de végétaux marins comme le foin de mer (Varech ou Zostère marine). Il a été utilisé car il attire très peu les insectes à cause de sa forte odeur, mais il ne s'emploie pas seul car très salé et donc hygroscopique. On le trouve souvent mélangé à du chanvre pour diminuer le risque de moisissure ${ }^{6}$. Avec les fibres textiles, c'est la catégorie de rembourrage que l'on a le plus de chance de trouver dans un spécimen datant d'avant 1950. Cette probabilité a été vérifiée grâce à l'étude de cas réalisée durant cette recherche : 1/3 des rembourrages étaient des tiges de graminées.

\section{Les dérivés du papier ou du textile.}

21 Le papier a été très peu utilisé car peu satisfaisant selon les taxidermistes. L'utilisation du papier mâché est plus fréquente que le papier non travaillé, car il se met en forme plus facilement. Il apparaît dès le XVIII ${ }^{e}$ siècle en Angleterre, puis s'étend en France au XIX ${ }^{\mathrm{e}}$ siècle. Cependant, durant l'étude du corpus du muséum d'Histoire naturelle de Neuchâtel, nous avons trouvé une feuille de papier entière possédant des inscriptions à la plume dans le corps d'un lion de mer du XIX ${ }^{e}$ siècle. Les noms d'oiseaux et les dimensions que nous avons déchiffrés nous laissent penser qu'il s'agit d'une page du carnet de note du préparateur, qui pourrait avoir été utilisée un peu "à la va-vite" pour terminer ce spécimen.

\section{Les dérivés du bois.}

Ils sont employés pour les réalisations les plus volumineuses car ce sont des matériaux très résistants, mais ils ont le désavantage de dégager des acides organiques plus ou moins concentrés en fonction des essences. Ils existent sous différentes formes : écorce, bois, liège et laine de bois. Les écorces et le bois sont sous forme de copeaux ou de sciure. Les copeaux de bois permettent de remplir le corps de façon assez grossière en restant léger, alors que la sciure a l'inconvénient d'alourdir le spécimen et de se vider lorsque la peau est percée. Le liège, surtout présent en Angleterre, a l'intérêt de se sculpter facilement et d'être très léger. Il est toujours utilisé aujourd'hui, mais sous forme de 
poudre mélangée à une résine acrylique. Enfin, la laine de bois aussi appelée frison de bois, apparue au XIX siècle et toujours utilisée aujourd'hui, se travaille de la même manière que la fibre de chanvre ou de lin, soit enroulée autour d'une structure porteuse. Le plus souvent les essences utilisées sont des résineux (plus particulièrement du mélèze qui a la réputation de faire fuir les insectes, ou de l'épicéa), mais on peut aussi trouver de l'orme, du tilleul, du saule ou du peuplier.

Feuille de papier trouvée au niveau de la couture ventrale d'un spécimen de lion de mer et présentant des écritures à la plume.

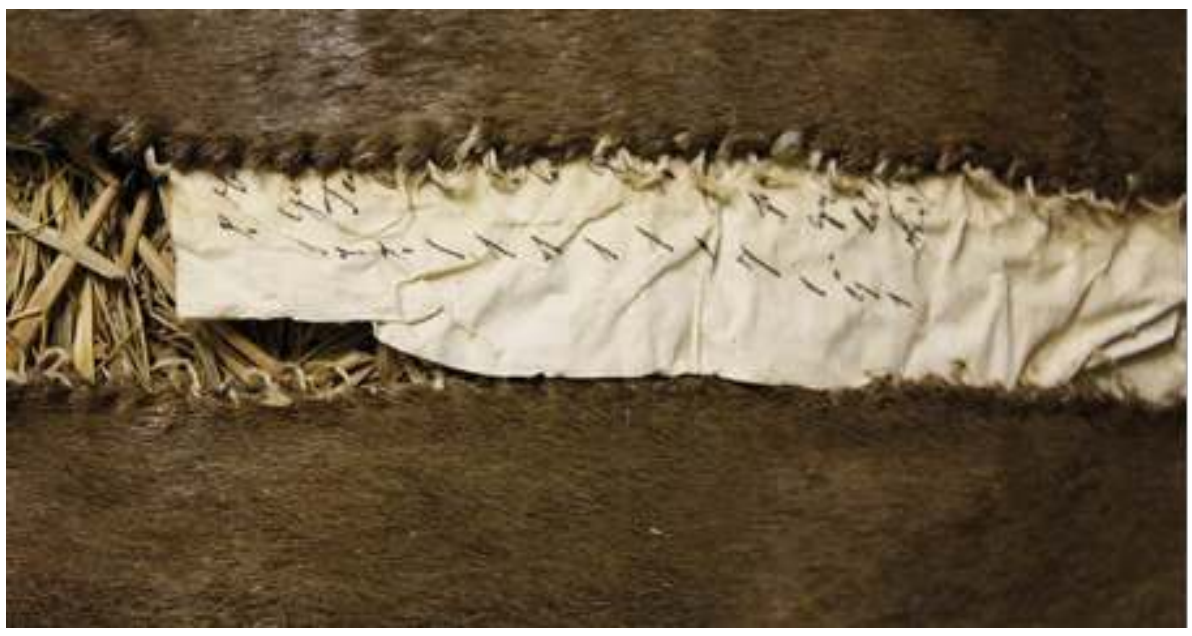

(c) HECR Arc G.B, 2015

Rembourrage en laine de bois, étoupe de chanvre et filasse

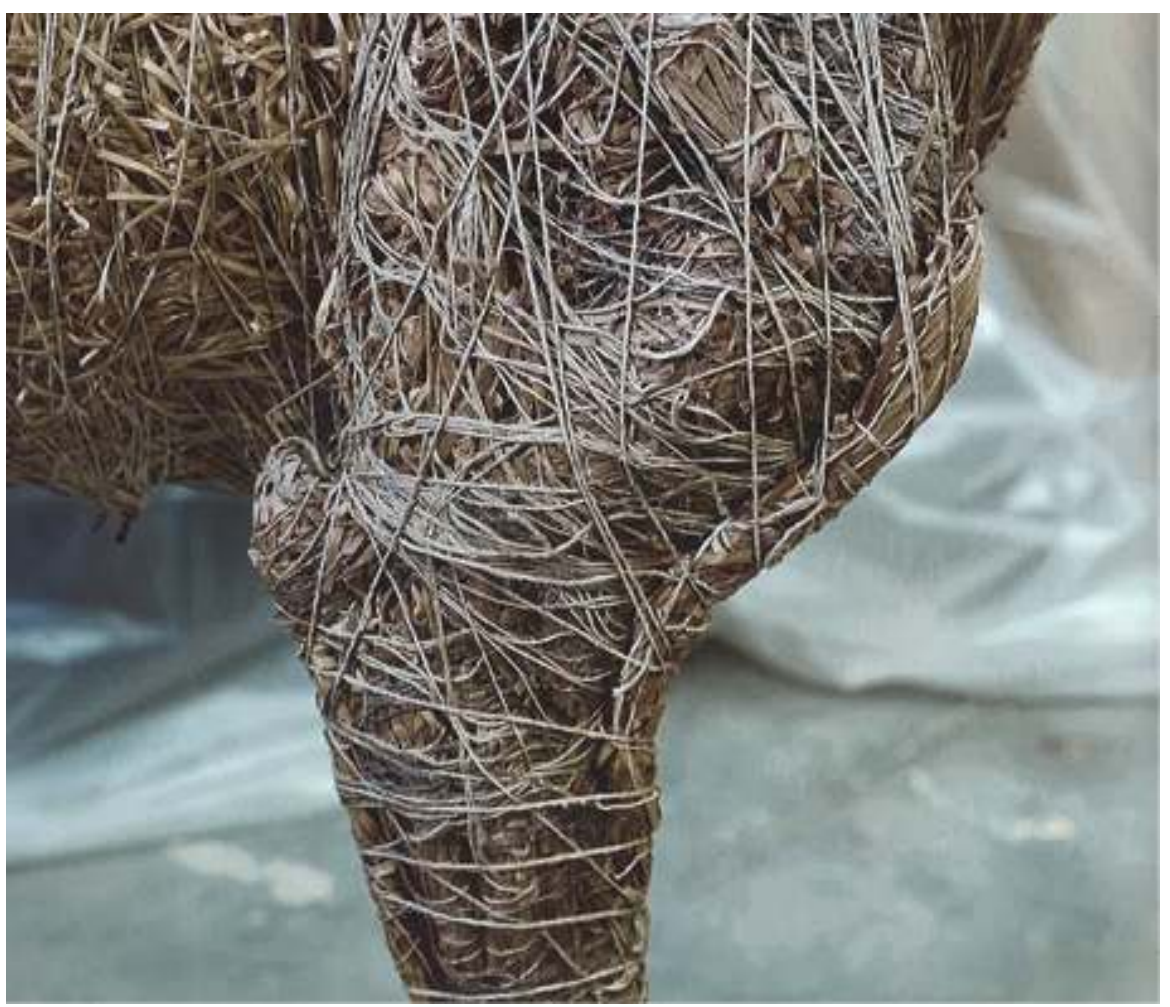

(c) Vekemans, 2014 


\section{Les matériaux d'origine animale}

I'argile (jusqu'au XXe siècle en Europe) sont les plus utilisés. Le plâtre est mentionné dans une quinzaine de références anglo-saxonnes selon l'étude de Williams et Hawks et par tous les auteurs français consultés. L'utilisation de ces matériaux est privilégiée pour les mammifères car la peau des oiseaux est trop fine pour supporter le poids du plâtre. Ils ont dans un premier temps été coulés directement dans le spécimen mais cela donnait un poids excessif au mannequin et cette technique a très vite été abandonnée. Occasionnellement, on peut rencontrer des mélanges de plâtre ou d'argile avec un liant autre que l'eau. L'abbé Manesse en 1787 parle par exemple d'un mastic, mélange de blanc d'Espagne en poudre et d'huile de noix, qu'il utilise pour réaliser le détail des lèvres et du museau. Ces matériaux à liant ont le désavantage d'être sujets à des variations dimensionnelles importantes car ils répondent aux changements hygrométriques externes. De plus, s'ils sont appliqués en couche trop mince et sans charge végétale, ils vont perdre toute cohésion en séchant, et vont s'effriter, entraînant l'affaissement du spécimen.

Sans liant. Dans la catégorie sans liant, on peut citer le sable. Son utilisation est limitée car il ne permet pas d'arrondir les formes de façon égale et naturelle et donne souvent un spécimen informe. De plus, comme la sciure, si la peau se déchire, il s'échappe du spécimen.

27 Les fibres. Pour finir, à partir de la deuxième moitié $\mathrm{du} \mathrm{XX}$ siècle, apparaissent les fibres minérales comme l'amiante. En mettant de côté leur toxicité, ces fibres nouvelles ont l'avantage d'être imputrescibles et très résistantes à la chaleur, à la friction, et aux acides mais leur utilisation est restée très limitée. Il n'existe que deux témoignages de l'utilisation de l'amiante'. 
Otarie à fourrure rembourrée au fil de soie.

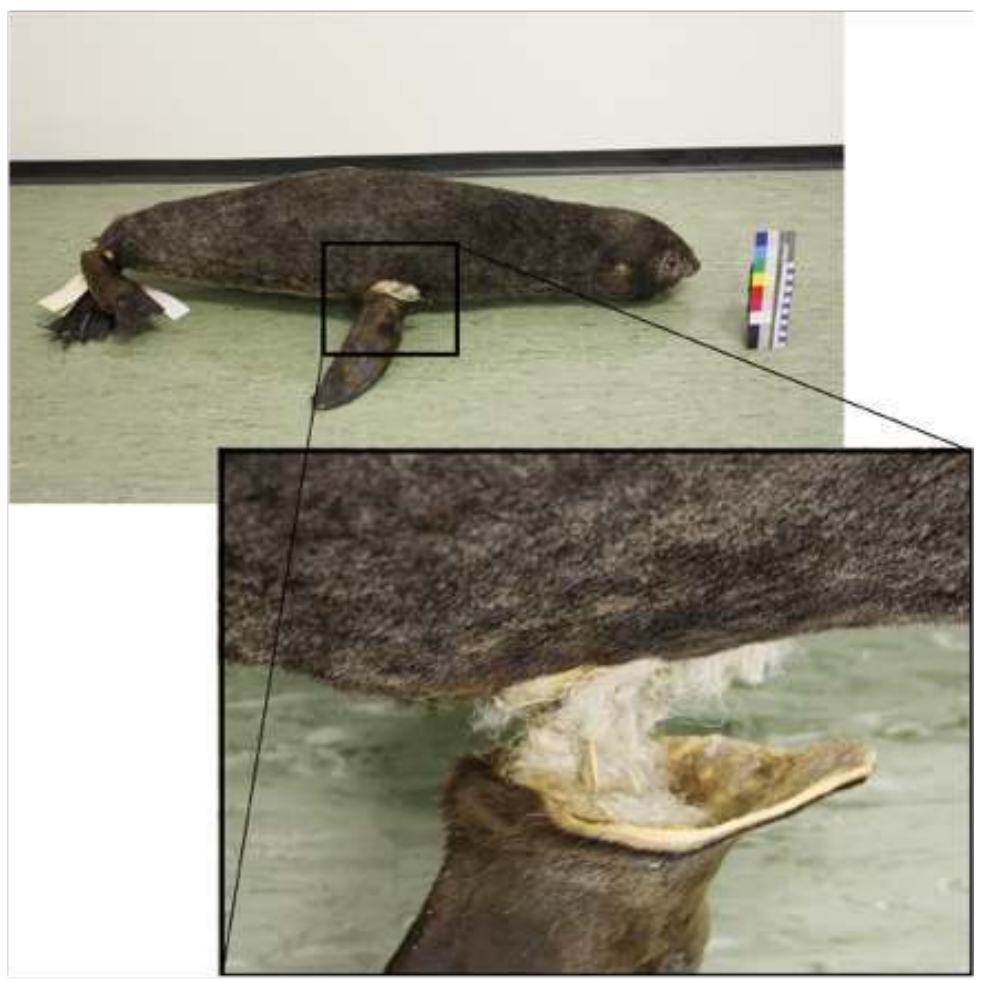

(c) HECR Arc G.B, 2015

Fissuration du plâtre d'un mannequin de chevreuil

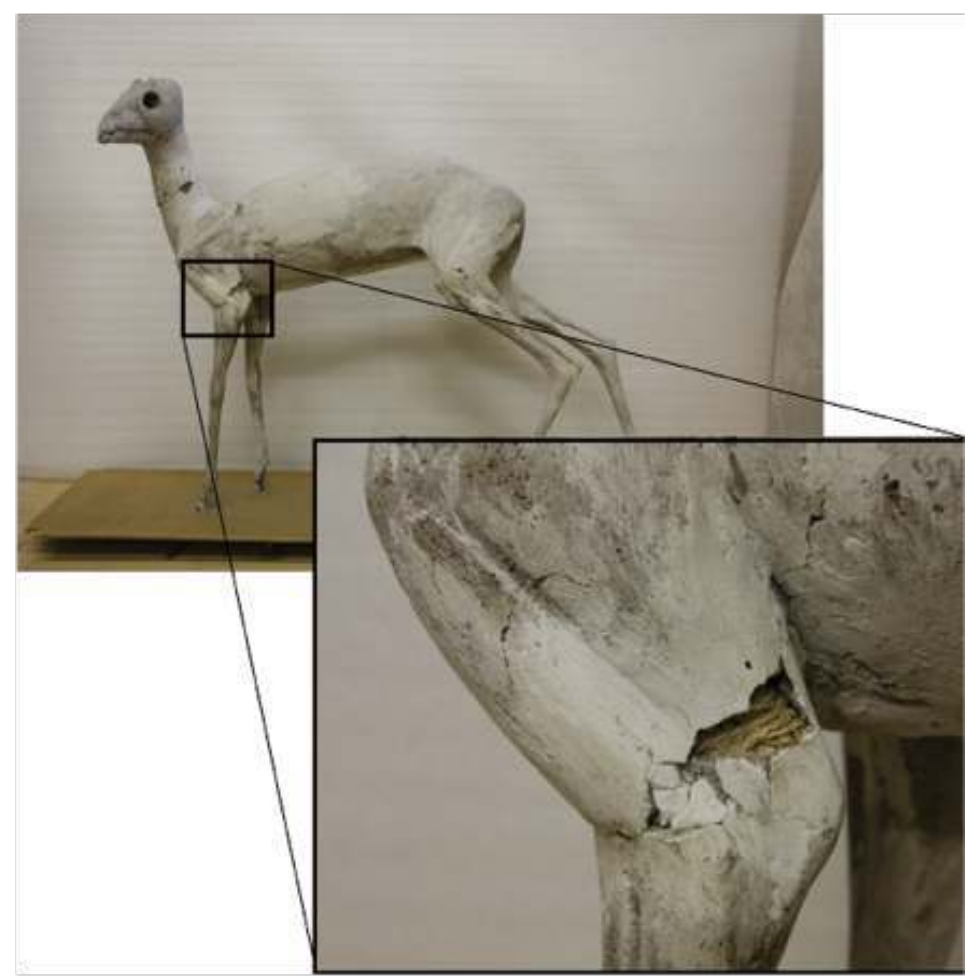

(c) HECR Arc G.B, 2015 


\section{Les matériaux d'origine synthétique}

À la fin des années 1960 apparaissent de nouveaux matériaux de synthèse qui remplacent peu à peu les traditionnels enchevêtrements de fibres. Ces matériaux se sont rapidement généralisés pour les montages des spécimens moyens à grands grâce à leur grande légèreté. Ils prennent la forme de mousses ou de résines aux densités différentes, agglomérées, extrudées, expansées ou coulées.

Jusque dans les années 1980, les polymères de synthèse utilisés dégageaient beaucoup d'acides ce qui a provoqué la perte de nombreux spécimens. Il reste très peu de témoignages de cette période et nous ne connaissons donc qu'une infime partie de ce qui a été utilisé comme rembourrage.

30 Le plastique qui a été le plus massivement utilisé est la mousse de Polyuréthane (PU) expansé de haute densité. Le remplissage à base de $\mathrm{PU}$ expansé est réalisé soit grâce à un mannequin préconçu, qui sera ensuite adapté à la morphologie du spécimen, soit en coulant le polymère dans un moule afin d'obtenir une forme non commercialisée et préparée sur mesure. Cette mousse donne un mannequin rigide et très léger, mais est chimiquement instable et libère des composés organiques volatiles (COV) lors de sa dégradation. De plus, la mise en forme de ce plastique dégage des gaz toxiques pour l'homme. Aujourd'hui le PU est déconseillé mais il est toujours utilisé pour les moyens et grands spécimens car il est rapide à mettre en forme et très léger ${ }^{8}$.

1 À l'heure actuelle, les Polystyrènes (PS) extrudés sont beaucoup utilisés. Pour limiter les échanges gazeux, on isole le mannequin en le recouvrant d'une toile de verre enrésinée. Cette méthode augmente la solidité du mannequin et sa stabilité chimique en créant une couche imperméable aux $\mathrm{COV}^{9}$. Mais malgré l'enrobage (appelé stratification), les professionnels ont encore peu de recul sur ce nouveau matériau, et il pourrait à terme poser des problèmes pour la conservation du spécimen. Il est par exemple sensible à la chaleur et aux solvants.

32 Un travail de recherche a récemment été entrepris par l'équipe de préparation du muséum de Toulouse afin de trouver un matériau synthétique stable qui pourrait remplacer le PS. C'est le Polyéthylène expansé de haute densité (PEHD) qui a été choisi car il est physiquement et chimiquement stable, ne craint pas les solvants et se met en forme facilement. Il est aujourd'hui le plus conseillé, et quotidiennement utilisé par le muséum selon la méthode qu'ils ont eux-mêmes mis au point ${ }^{10}$.

Référencement des matériaux de rembourrage, résultat de la recherche

Ce tableau présente l'intégralité des matériaux de rembourrage trouvés lors de la recherche (documentation et étude de cas confondues). Une datation approximative d'utilisation est précisée lorsqu'elle est connue. 


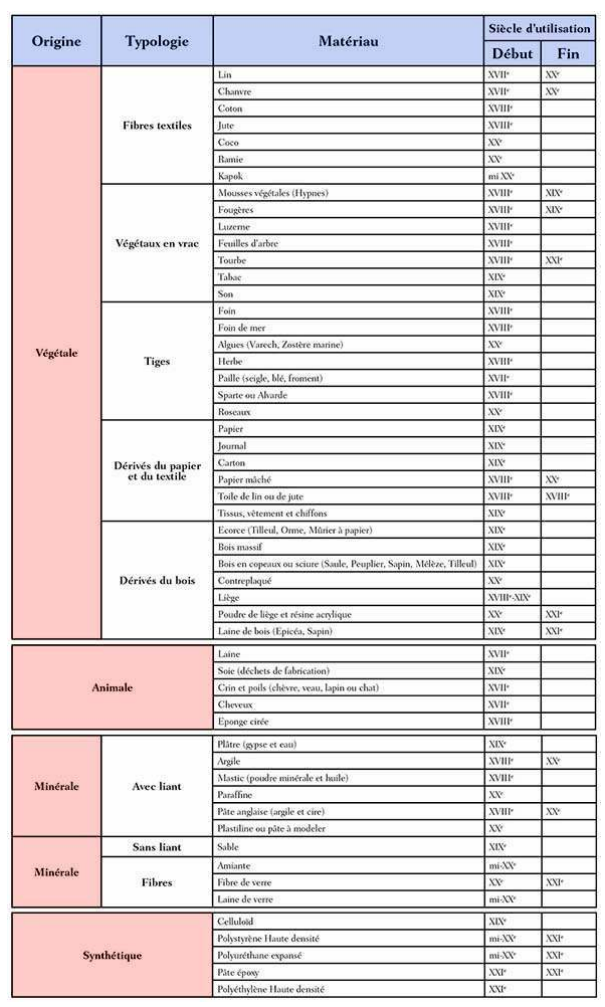

\section{Conclusion}

Durant l'étude, nous avons pu nous rendre compte de la très grande variété de ces matériaux. En effet, nous avons inventorié 54 familles de rembourrages différents, ce qui ne représente probablement que la partie émergée de l'iceberg. En témoigne notamment la découverte du fil de soie, de la fibre de coco (voir tableau) et de la feuille de carnet de note dans les spécimens naturalisés du corpus étudié qui ne figuraient pas dans les recherches documentaires préliminaires. Puis, grâce à l'identification des rembourrages du corpus, nous pouvons entrevoir la proportion des matériaux utilisés : $1 / 3$ de fibres textiles, $1 / 3$ de tiges de graminées, et $1 / 3$ de matériaux divers (mousses, tourbe, liège, papier...).

Cette recherche a mis en valeur le facteur expérimental de la pratique de la taxidermie et le côté artisanal de la profession qui a parfois amené les préparateurs à utiliser les matériaux qu'ils avaient "sous la main". Cette très grande variété de matériaux et cette absence de "norme" amène une confusion certaine et nous empêche - sauf exception d'utiliser la nature du rembourrage comme moyen de datation précis de la fabrication du spécimen. En revanche, il est possible dans certains cas de les utiliser comme marqueur géographique. Par exemple, la fibre de coco, identifiée dans une tortue de mer des Seychelles, nous indique une forte probabilité que le spécimen ait été préparé sur place.

S'ils sont des sources d'informations historiques et techniques non négligeables, ces rembourrages apparaissent aussi comme un facteur de dégradation supplémentaire et peuvent provoquer des fissurations ou déchirures de la peau, des distensions des coutures, des gonflements, ou des affaissements. Ainsi, en connaissant la nature du rembourrage, on pourra anticiper ou diagnostiquer ces dégradations, mais également 
donner aux Naturalia des conditions de conservation adaptées sans négliger les matériaux internes, permettant ainsi une meilleure conservation sur le long terme.

\section{BIBLIOGRAPHIE}

Abbé Manesse. Traité sur la manière d'empailler et de conserver les animaux, les pelleteries et les laines. Paris : Guillot, 1787, 190 p.

Aïello, B. L'utilisation des Polyéthylènes en taxidermie, La Lettre de l'OCIM, n¹63, 2016, pp. 15-21.

Boitard, M. Nouveau manuel complet du naturaliste préparateur, ou l'art d'empailler les animaux, de conserver les végétaux et les minéraux, de préparer les pièces d'anatomie normale et pathologique. Paris : Librairie encyclopédique de Roret, 1853, 452 p.

Cuisin, J. Fragilité des naturalia, risques non fonctionnels des collections de mammifêres et d'oiseaux, CRBC, Vol. 22, 2004, pp. 11-26.

Fève, P. Brochure d'éducation nouvelle populaire : naturalisations. Cannes : Éditions de l'école moderne française, 1951, $32 \mathrm{p}$.

Larsen, H. La taxidermie moderne. Éléments de la technique pour la préparation et le montage des animaux. Genève : Éditions de la Frégate, 1945, 138 p.

Palaus, X. La taxidermie : outillage, substances conservatrices, préparation des oiseaux, des mammifères, des reptiles, des insectes, conservation des collections. Paris : De Vecchi, 1983, 140 p.

Péquignot, A. Histoire de la Taxidermie en France (1769-1928) - Étude des facteurs de ses évolutions techniques et conceptuelles, et ses relations à la mise en exposition du spécimen naturalisé. Thèse du Muséum national d'Histoire naturelle, 2002, 367 p.

Péquignot, A. The history of taxidermy : clues for preservation, A journal for Museum and Archives Professionals, vol. 2, n³, 2006, pp. 245-255.

Swainson, W. Taxidermy, bibliography and biography. London : Printed for Longman, Orme, Brown, Green \& Longmans, 1840, $404 \mathrm{p}$.

Thiney, J. Mort ou vif. Chronique d'une taxidermie contemporaine. Paris : Éditions de la Martinière, Muséum national d'Histoire naturelle, 2014, 192 p.

Williams, S.-L. et Hawks, C.-A. History of preparation materials used for recent mammal specimens. Mammal collection management, 1987, pp. 21-49.

\section{NOTES}

1. Péquignot, 2002.

2. Péquignot, 2006.

3. Techniques de Larsen et Fève dans les années 1950. Une colle (mélange de gomme arabique et de sucre blanc) était déjà utilisée par Swainson en 1840 mais uniquement pour le montage final. 
4. Critiqué notamment par l'abbé Manesse qui n'utilise pas de coton pour ses préparations en 1787.

5. Technique utilisée par Henry Larsen en 1945, p. 59.

6. En 1845, Boitard préconise de le sécher préalablement au four pour tuer les parasites, de même que le foin et la paille.

7. Palaus, 1983, p. 13 ; Williams et Hawks, 1987, p. 24.

8. Cuisin, 2004, p. 21.

9. Technique utilisée par Jack Thiney et décrite dans son livre Mort ou Vif. Chronique d'une taxidermie contemporaine.

10. Aïello, B. L'utilisation des Polyéthylènes en taxidermie, La Lettre de l'OCIM, n¹63, 2016, pp. $15-21$.

\section{RÉSUMÉS}

Cette étude historique sur la nature des rembourrages, leur référencement et leur mise en forme a été réalisée afin de compléter les connaissances sur les matériaux constitutifs des spécimens naturalisés et sur leur histoire. L'objectif était de faciliter le travail des restaurateurs et des conservateurs dans l'optique d'améliorer la préservation des spécimens sur le long terme.

\section{INDEX}

Mots-clés : taxidermie, conservation

\section{AUTEUR}

\section{GAËLLE DUFFAU}

Diplômée en conservation de la Haute École de Conservation-Restauration-Arc de Neuchâtel et collaboratrice en conservation préventive indépendante (France et Suisse)

duffau.gaelle@gmail.com 